\title{
Antiemetic Prophylaxis for Chemoradiotherapy- induced Nausea and Vomiting (C-RINV) in Locally Advanced Head and Heck Squamous Cell Carcinoma: a Prospective Phase $₫$ Trial
}

\section{Zekun Wang}

Cancer Hospital Chinese Academy of Medical Sciences

Wenyang Liu

Cancer Hospital Chinese Academy of Medical Sciences

Jianghu Zhang

Cancer Hospital Chinese Academy of Medical Sciences

\section{Xuesong Chen}

Cancer Hospital Chinese Academy of Medical Sciences Jingbo Wang

Cancer Hospital Chinese Academy of Medical Sciences

\section{Kai Wang}

Cancer Hospital Chinese Academy of Medical Sciences

\section{Yuan Qu}

Cancer Hospital Chinese Academy of Medical Sciences

\section{Xiaodong Huang}

Cancer Hospital Chinese Academy of Medical Sciences

\section{Jingwei Luo}

Cancer Hospital Chinese Academy of Medical Sciences

\section{Jianping Xiao}

Cancer Hospital Chinese Academy of Medical Sciences

\section{Guozhen Xu}

Cancer Hospital Chinese Academy of Medical Sciences

\section{Li Gao}

Cancer Hospital Chinese Academy of Medical Sciences Junlin Yi

Cancer Hospital Chinese Academy of Medical Sciences

Ye Zhang ( $\nabla$ drzye1983@163.com )

Chinese Academy of Medical Sciences Cancer Institute and Hospital https://orcid.org/0000-00026052-0708 
Research

Keywords: Concurrent chemoradiotherapy, Head and neck, Nausea, Emesis, Antiemetic prophylaxis

Posted Date: October 1st, 2021

DOI: https://doi.org/10.21203/rs.3.rs-948378/v1

License: (c) (i) This work is licensed under a Creative Commons Attribution 4.0 International License. Read Full License 


\section{Abstract}

Background There is sparse research reporting effective interventions for preventing nausea and emesis caused by concurrent chemoradiotherapy (CCRT) in locally advanced head and neck squamous cell carcinoma (LA-HNSCC). This phase $\otimes$ trial was conducted to provide the direct evidence for the current practice of prescribing antiemetic in patients with LA-HNSCC receiving CCRT.

Methods Treatment-naïve LA-HNSCC patients received intensity-modulated radiotherapy with concomitant cisplatin $100 \mathrm{mg} / \mathrm{m}^{2}$ every 3 weeks for two cycles. All patients were given orally aprepitant $125 \mathrm{mg}$ once on $\mathrm{d} 1$, then $80 \mathrm{mg}$ once on d2-5; ondansetron $8 \mathrm{mg}$ once on $\mathrm{d} 1$; and dexamethasone $12 \mathrm{mg}$ once on $\mathrm{d} 1$, then $8 \mathrm{mg}$ on $\mathrm{d} 2-5$. The primary endpoint was complete response (CR). Pursuant to $\delta=0.2$ and $a=0.05$, the expected $C R$ rate was $80 \%$.

Results A total of 43 patients with LA-HNSCC were enrolled. The median age was 53 years old, and $86.0 \%$ were male. All patients received radiotherapy and $86.0 \%$ of patients completed both cycles as planned. The overall CR rate was $86.0 \%$ (95\% Cl: 72.1-94.7). The CR rates for cycles 1 and 2 were $88.4 \%$ (95\% Cl: $74.9-96.1)$ and $89.2 \%$ (95\% Cl: 74.6-97.0). The complete protection rate in the overall phase was $72.1 \%$ (95\% Cl: 56.3-84.7). The emesis-free response and nausea-free response in overall phase were $88.4 \%$ (95\% Cl: 74.9-96.1) and 60.5\% (95\% Cl: 44.4-75.0), respectively. The adverse events related to antiemetics were constipation (65.1\%) and hiccups (16.3\%), but both were grade 1-2. There was no grade 4 or 5 treatment-related adverse event with antiemetic usage.

Conclusion The addition of aprepitant into ondansetron and dexamethasone provided effective protection from nausea and emesis in patients with LA-HNSCC receiving radiotherapy and concomitant high-dose cisplatin chemotherapy. Randomised phase 3 studies are required to further define the potential role of $\mathrm{NK}_{1} \mathrm{RA}$ in chemoradiotherapy setting.

Trial registration: ClinicalTrials.gov, number NCT03572829. Registered 28 June 2018, https://clinicaltrials.gov/ct2/show/NCT03572829?term=NCT03572829\&draw=2\&rank=1.

\section{Background}

Amongst the background of advances in new antiemetic agents, the efficacy of antiemetics in preventing chemotherapy-induced nausea and vomiting (CINV) has been substantially improved ${ }^{1}$. Aprepitant was the first Neurokinin-1 receptor antagonist $\left(\mathrm{NK}_{1} \mathrm{RA}\right)$ that block the binding of substance $P$ to prevent emesis. The addition of $\mathrm{NK}_{1} \mathrm{RAs}$ improved complete response (no emesis and no rescue medication) by 8-20\% after highly emetogenic chemotherapy (HEC) ${ }^{2}$. The current Multinational Association of Supportive Care in Cancer/ European Society for Medical Oncology (MASCC /ESMO) and NCCN guidelines also recommend a triple regimen consisting of 5-HT3RA, dexamethasone and $\mathrm{NK}_{1} \mathrm{RAs}$ to prevent nausea and vomiting induced by HEC, e.g., cisplatin ${ }^{3}$. Conversely, little focus has centred on the antiemetic prophylaxis in radiotherapy. As a result, radiation-induced nausea and vomiting (RINV) is often 
underestimated by clinicians ${ }^{4}$. A cross-sectional study demonstrated one third of patients experiencing RINV would like additional treatment ${ }^{5}$. Nevertheless, evidence for the guideline recommendations is scarce, and little is known about the combination and optimal duration of antiemetic agents ${ }^{6}$. In chemoradiotherapy setting, RINV can be exacerbated by concomitant chemotherapy to higher levels, which has been confirmed by prior studies, though the pathophysiological mechanism is not well understood $^{7-9}$.

With regard to locally advanced head and neck squamous cell carcinoma (LA-HNSCC), cisplatin-based concurrent chemoradiotherapy (CCRT) remains the standard treatment modality. However, nausea and vomiting induced by CCRT are common toxicities, with deleterious effects on treatment compliance and quality of life. Several prospective studies have demonstrated that the incidence of nausea and vomiting during cisplatin-based CCRT are $48 \%-66 \%$ and $30 \%-47 \%{ }^{10-13}$. Thus, there is a pressing need for optimization of antiemetic treatment to address chemoradiotherapy-induced nausea and vomiting (CRINV). Recent evidence has emerged that $\mathrm{NK}_{1} \mathrm{RAs}$ improve the control of C-RINV in certain tumors. To date, only five published prospective studies have reported the efficacy and safety of $\mathrm{NK}_{1} \mathrm{RAs}$ for the prophylaxis of C-RINV, two of which focused on cervical cancer, and the remainder included multiple-site

tumors ${ }^{14-18}$. The above studies demonstrated the superiority of $\mathrm{NK}_{1} \mathrm{RAs}$ in the prevention of nausea and vomiting as well as their high tolerance, yet whether different tumors, various chemotherapy regimens, as well as different radiation schedules would yield similar results remains unknown.

In our preliminary trial, 13 patients with LA-HNSCC receiving CCRT with triweekly cisplatin were administrated with triple antiemetic regimen consisting of $\mathrm{NK}_{1} \mathrm{RA}$ (aprepitant), $5-\mathrm{HT}_{3} \mathrm{RA}$ (ondansetron) and dexamethasone. The results highlight only 1 case developed vomiting and 2 cases required rescue antiemetic treatment. No significant adverse event was observed. On the basis of the above results, a prospective trial was conducted to explore whether the addition of aprepitant could provide effective protection against C-RINV in patients with LA-HNSCC.

\section{Materials And Methods}

\section{Study design and participants}

This study was investigator initiated and designed as a prospective single arm, open label phase $₫$ trial to evaluate the efficacy and safety of aprepitant combined with ondansetron and dexamethasone for the prevention of nausea and vomiting induced by concurrent chemoradiotherapy in LA-HNSCC. All patients were fully informed about the study and signed informed consent. The study was conducted in accordance with the Declaration of Helsinki and the international standards of Good Clinical Practice and approved by the local ethics committee.

The eligible patients were aged 18 to 70 years old with histologically confirmed squamous cell carcinoma of the head and neck (nasopharynx, oral cavity, oropharynx, hypopharynx, larynx, nasal cavity and 


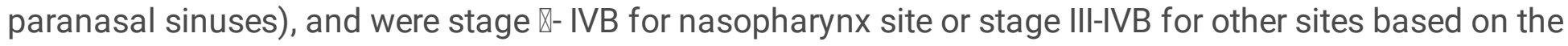
American Joint Committee on Cancer 7th Edition (AJCC 7th ). Patients had the Eastern Cooperative Oncology Group (ECOG) performance status 0-1; had adequate organ and bone marrow function; must have been able to swallow tablets; had the life expectancy of at least 12 weeks; and would receive CCRT as planned after multi-disciplinary team (MDT) discussion. Fertile male and female patients volunteered to use effective contraception during the study and within 90 days of the last medication. The exclusion criteria included other medical histories of malignancy apart from non-melanoma skin cancer, cervical carcinoma in situ, and early-stage cured prostate cancer; nausea and emesis occurred 24 hours before the start of CCRT; corticosteroid or benzodiazepines used; any medicine which affected metabolism through drug-metabolising enzymes CPY3A4 and CYP2D6 except for nighttime sedatives; severe cardiovascular, pulmonary, diabetes, mental and other diseases; perinatal women or rejection of taking contraception during treatment; drug and/or alcohol addiction; symptomatic brain metastasis; gastrointestinal obstruction; hypocalcemia or any other conditions that could provoke emesis.

\section{Procedures}

Intensity-modulated radiotherapy (IMRT) using simultaneously integrated boost was administered to all patients. The target delineation and dose prescription followed the principles of radiotherapy for head and neck cancer at our center ${ }^{19}$. The delineation of normal organs adhered to the international consensus on delineation of organs at risk in the head and neck regions ${ }^{20}$, and the standard of dose limitation referred to the QUANTEC. Concurrent chemotherapy was administrated with cisplatin 100 $\mathrm{mg} / \mathrm{m}^{2}$ (given evenly on days 1-3) every 3 weeks for two cycles. All patients received hydration and diuretic therapy during the administration of cisplatin. The chemotherapy-induced toxicities had to be restored to grade 0-2 prior to the start of the subsequent cycle. The chemotherapy was terminated if the toxicities were not restored to grade $0-2$ within 14 days of the beginning of the next cycle. If grade 4 hematological toxicities or $\geq$ grade 3 non-hematological toxicities occurred in the previous cycle, the dose of subsequent cycle had to be reduced by $25 \%$.

The recruited patients were subjected to unified antiemetic treatment (Fig. 1). The doses, duration and scheduling of antiemetics in our study basically followed the MASCC/ESMO guidelines for multiple-day cisplatin chemotherapy. ${ }^{21} \mathrm{~A}$ combination of ondansetron, dexamethasone and aprepitant was applied for acute nausea and vomiting, dexamethasone and aprepitant for delayed nausea and vomiting. Aprepitant was administrated orally once daily for the 2 days after chemotherapy (days 4-5). The antiemetic regimen was maintained in the subsequent cycle until chemotherapy was stopped or severe drug-related side effects occurred. Rescue antiemetic therapy was permitted for grade 3 nausea (National Cancer Institute Common Toxicity Criteria for Adverse Events, NCI CTCAE, version 4.0) or poor antiemetic effects (criteria: emesis $>2$ times/24 hours, and continuous vomiting within 5 minutes is regarded as one time).

\section{Assessment}

Patients were obligated to complete daily diaries per cycle that recorded nausea, vomiting or dry retches including the time, frequency, degree or score, and application of rescue medication. The weekly 
evaluation of treatment-related toxicities was predicated on $\mathrm{NCI}$ CTCAE version 4.0. To assess the degree of nausea and vomiting, the MASCC Antiemesis Tool (MAT) scale was completed respectively by patients during chemotherapy and during the period from the 2 nd day to 4th day after the last administration of cisplatin. At baseline, on day 7 of the first cycle and the second cycle, the Functional Living Index-Emesis (FLIE) questionnaire was employed to evaluate the effect of nausea and emesis on the quality of life. The overall quality of life was assessed each week during treatment (week 1-7) by using the questionnaires of the European Organization for Research and Treatment of Cancer (EORTC), Quality of Life Core 30 items (QLQ-C30) version 3.0 and Quality of Life Head and Neck 35 items (QLQ-H\&N35) version 1.0.

\section{Outcomes}

The primary endpoint was complete response (CR) defined as no emesis and no use of rescue therapy during concurrent chemoradiotherapy. The secondary endpoints were as follows: CR for each cycle; complete protection (CP), denoted as no emesis, no rescue medication and no more than mild nausea (grade 1 premised on CTCAE 4.0); emesis-free response, which refers to no vomiting or dry retches during treatment; nausea-free response, signified as no nausea during treatment; and treatment-related toxicities. Other secondary endpoints consisted of the degree of nausea and vomiting evaluated by the MAT scale, as well as the quality of life using the FLIE, EORTC QLQ-C30 and QLQ-H\&N35 questionnaires (results not reported here).

\section{Statistical analysis}

The Simon two-stage design was adopted to estimate the sample size ${ }^{22}$. Pursuant to $\delta=0.2$ and $a=$ 0.05 , the expected $C R$ rate was $80 \%$. If the CR was less than $60 \%$, the study protocol was declared invalid. In the first stage, 17 patients were enrolled. If more than 13 patients achieved CR, 26 patients would remain enrolled.

Demographic and clinical characteristics were outlined using median (range) for continuous variables and frequency (percentage) for categorical variables. The percentages of patients with CR or CP, emesisfree response, and nausea-free response for every cycle and overall phase were estimated along with $95 \%$ confidence intervals (Cls). The corresponding $95 \% \mathrm{Cls}$ were calculated using the Binomial test of onesample nonparametric tests. The treatment-related toxicities and adverse events were summarised as frequency (percentage). Statistical analyses were conducted using SPSS software (version 26.0; SPSS, Inc).

\section{Results}

Between March 2018 and January 2020, 16 of 17 patients recruited in stage 1 achieved CR and thereafter 26 patients were further enrolled in stage 2 . Baseline characteristics are reported in Table 1 . The median age was 53 years old (range: 18-66), and $86.0 \%$ were male. 40 patients in total were treated with definitive CCRT, while the remaining 3 patients who were to receive planned definitive CCRT were re-examined and the efficacy thereof evaluated when the cumulative PTV dose reached around 50Gy, followed by conversion to surgery after MDT discussion. The median radiation dose was 69.96Gy (range: 53- 
73.92Gy). All patients received radiotherapy and the first cycle of cisplatin, while 6 patients terminated the subsequent cycle of chemotherapy. Among the 37 patients who received the second cycle of chemotherapy, different degrees of delayed chemotherapy occurred in 21 (56.8\%) patients (median: 7 days; range: 2-14 days). The reasons for delay or discontinuation are displayed in Table 2. Cisplatin dose changes were reported in 11 (29.7\%) of 38 patients during the second cycle. All patients completed antiemetic treatment as planned. 
Table 1

Baseline demographic and clinical characteristics

\section{Characteristics}

Age, years

Gender

Male

Female

ECOG performance status

0

1

Tumor location

Nasopharynx

Oropharynx

Hypopharynx

Larynx

Paranasal sinuses

TNM classification (AJCC 7th)

$\begin{array}{ll}\square & 3(7.0 \%) \\ \square & 16(37.2 \%) \\ \square \mathrm{A} & 17(39.5 \%) \\ \square \mathrm{B} & 7(16.3 \%)\end{array}$

Completed cycles

One cycle

Two cycles

Total dose of cisplatin, $\mathrm{mg}$

Dose of cycle 1

Dose of cycle 2

Data are $n, n(\%)$, or median (range). ECOG=Eastern Cooperative Oncology Group. CCRT = Concurrent Chemoradiotherapy.
$N=43$

$53(18-66)$

$37(86.0 \%)$

$6(14.0 \%)$

$5(11.6 \%)$

38 (88.4\%)

23 (53.5\%)

9 (20.9\%)

8 (18.6\%)

$1(2.3 \%)$

$2(4.7 \%)$

43 (100\%)

37 (86.0\%)

300 (150-360)

150 (120-180)

150 (90-180) 
Table 2

Reasons for chemotherapy delay or discontinuation

\begin{tabular}{|ll|}
\hline Reasons & Numbers \\
\hline Chemotherapy delay & 21 \\
\hline Hematologic toxicities & 12 \\
\hline Hepatic function damage & 3 \\
\hline Patient autonomy & 3 \\
\hline Gastrointestinal reactions & 2 \\
\hline Asthenia/fatigue & 1 \\
\hline Chemotherapy discontinuation & 6 \\
\hline CoVID-19 pandemic & 2 \\
\hline Thromboembolic event & 1 \\
\hline Hepatic dysfunction & 1 \\
\hline Malnutrition & 1 \\
\hline Patient refusal & 1 \\
\hline
\end{tabular}

The overall CR rate of this study achieved $86.0 \%$ (95\% Cl: 72.1\%-94.7\%), exceeding the expected CR rate. Table 3 lists the secondary efficacy endpoints. The CR rate for cycle 1 was $88.4 \%$ (95\% Cl: 74.9\%-96.1\%), while there was a slightly higher $\mathrm{CR}$ rate $(89.2 \%, 95 \% \mathrm{Cl}: 74.6 \%-97.0 \%)$ for cycle 2 . The CP rate in the overall phase was $72.1 \%$ (95\% Cl: $56.3 \%-84.7 \%)$, while the data of cycle 1 and cycle 2 were $76.7 \%$ (95\% Cl: $61.4 \%-88.2 \%)$ and $86.5 \%$ (95\% Cl: $71.2 \%-95.5 \%)$, respectively. 
Table 3

Secondary efficacy endpoints

\begin{tabular}{|c|c|c|}
\hline & No. of patients (percentage) & $95 \% \mathrm{Cl}$ \\
\hline \multicolumn{3}{|l|}{ Complete response } \\
\hline Days 1-3 of cycle 1 & $39(90.7 \%)$ & $77.9 \%-97.4 \%$ \\
\hline Days 1-7 of cycle 1 & $38(88.4 \%)$ & $74.9 \%-96.1 \%$ \\
\hline Days $1-3$ of cycle 2 & $34(91.9 \%)$ & $78.1 \%-98.3 \%$ \\
\hline Days 1-7 of cycle 2 & $33(89.2 \%)$ & $74.6 \%-97.0 \%$ \\
\hline \multicolumn{3}{|c|}{ Emesis-free response } \\
\hline Days $1-3$ of cycle 1 & $40(93.0 \%)$ & $80.9 \%-98.5 \%$ \\
\hline Days 1-7 of cycle 1 & $39(90.7 \%)$ & $77.9 \%-97.4 \%$ \\
\hline Days $1-3$ of cycle 2 & $34(91.9 \%)$ & $78.1 \%-98.3 \%$ \\
\hline Days 1-7 of cycle 2 & $34(91.9 \%)$ & $78.1 \%-98.3 \%$ \\
\hline \multicolumn{3}{|c|}{ Nausea-free response } \\
\hline Days 1-3 of cycle 1 & $30(69.8 \%)$ & $53.9 \%-82.8 \%$ \\
\hline Days 1-7 of cycle 1 & $26(60.5 \%)$ & $44.4 \%-75.0 \%$ \\
\hline Days $1-3$ of cycle 2 & $32(86.5 \%)$ & $71.2 \%-95.5 \%$ \\
\hline Days 1-7 of cycle 2 & $29(78.4 \%)$ & $61.8 \%-90.2 \%$ \\
\hline \multicolumn{3}{|l|}{ Complete protection } \\
\hline Days 1-3 of cycle 1 & $36(83.7 \%)$ & $69.3 \%-93.2 \%$ \\
\hline Days 1-7 of cycle 1 & $33(76.7 \%)$ & $61.4 \%-88.2 \%$ \\
\hline Days $1-3$ of cycle 2 & $33(89.2 \%)$ & $74.6 \%-97.0 \%$ \\
\hline Days $1-7$ of cycle 2 & $32(86.5 \%)$ & $71.2 \%-95.5 \%$ \\
\hline
\end{tabular}

The emesis-free response in overall phase achieved 88.4\% (95\% Cl: 74.9\%-96.1\%). The emesis-free response rates for cycle 1 and 2 were 90.7\% (95\% Cl: 77.9\%-97.4\%) and 91.9\% (95\% Cl: 78.1\%-98.3\%), respectively. Of the five patients with emesis, grade 2 emesis occurred in three patients, the remaining two patients experienced grade 1 emesis, and all patients did not receive rescue antiemetics. The median time from cisplatin administration to emesis was the 3rd day (day 1-day 6), while the median duration time of emesis was 2 days (1-6 days). 
The nausea-free response in the overall phase was $60.5 \%$ (95\% Cl: $44.4 \%-75.0 \%)$. The nausea-free response rate for cycle 1 also achieved $60.5 \%$ (95\% Cl: 44.4\%-75.0\%), whereas the incidence of no nausea for cycle 2 increased by nearly $18 \%$ (78.4\%; $95 \%$ Cl: $61.8 \%-90.2 \%)$. The distribution of patients with different grades of nausea appertaining to the nausea visual analog scale (NVAS) was reported in the Fig. 2 (1-3 points: 7 cases; 4-6 points: 7 cases; 7-10 points: 3 cases). The median score of nausea founded on the NVAS was 4 points (range: 1-10). Regarding the patients with nausea, the same one patient developed severe nausea (NVAS score up to 10 points) in both cycles and was administrated with rescue treatment. The median time of the nausea episode was the 2 nd day (day 1-day 6), while the median duration time of nausea was 3 days (1-7days).

Grade 1-2 adverse events that presented in at least $10 \%$ patients included dermatitis, mucositis, dry mouth, leucopenia, anaemia, hepatic dysfunction, appetite loss, constipation, and hiccups (Table 4). The most frequent grade 3 adverse events were mucositis (20.9\%) and leucopenia (14.0\%). Grade 3 dermatitis, hepatic dysfunction, and appetite loss were less than $5 \%$, and no grade 4 or 5 adverse event was recorded. The adverse events related to antiemetics were constipation (65.1\%) and hiccups (16.3\%), but both were grade 1-2. Further, no infusion site infection or allergic reaction emerged during treatment.

Table 4

Treatment-related toxicities

\begin{tabular}{|llll|}
\hline Toxicities & Grade 1 & Grade 2 & Grade 3 \\
\hline Dermatitis & $28(65.1 \%)$ & $14(32.6 \%)$ & $1(2.3 \%)$ \\
\hline Mucositis & $13(30.2 \%)$ & $18(41.9 \%)$ & $9(20.9 \%)$ \\
\hline Dry mouth & $14(32.6 \%)$ & $29(67.4 \%)$ & 0 \\
\hline Leucopenia & $13(30.2 \%)$ & $14(32.6 \%)$ & $6(14.0 \%)$ \\
\hline Anaemia & $11(25.6 \%)$ & $2(4.7 \%)$ & 0 \\
\hline Hepatic dysfunction & $8(18.6 \%)$ & $3(7.0 \%)$ & $2(4.7 \%)$ \\
\hline Loss of appetite & $18(41.9 \%)$ & $10(23.3 \%)$ & $2(4.7 \%)$ \\
\hline Constipation & $15(34.9 \%)$ & $13(30.2 \%)$ & 0 \\
\hline Hiccups & $4(9.3 \%)$ & $3(7.0 \%)$ & 0 \\
\hline $\begin{array}{l}\text { Data are } n \text { (\%). Incidences of grade 1-2 adverse events in at least 10\% of patients and all grade 3 } \\
\text { adverse events. No grade 4 or 5 adverse event was observed. }\end{array}$ \\
\hline
\end{tabular}

\section{Discussion}

To the best of our knowledge, this is the first prospective trial to evaluate the efficacy and safety of a NK receptor antagonist (aprepitant) combined with ondansetron and dexamethasone to prevent nausea and vomiting for patients with HNSCC receiving triweekly cisplatin chemoradiotherapy. The primary endpoint 
met the target, with the overall CR rate achieving $86.0 \%$, revealing that the triple antiemetic regimen provided effective protection against chemoradiotherapy-induced nausea and vomiting in patients with LA-HNSCC. Prior research has indicated that the addition of an $\mathrm{NK}_{1} \mathrm{RA}$ into $5-\mathrm{HT}_{3} \mathrm{RA}$ and dexamethasone improves antiemetic efficacy in patients with cervical cancer receiving radiotherapy with weekly cisplatin chemotherapy 17,23 . Similar results have now been extended to patients with HNSCC receiving IMRT and triweekly cisplatin chemotherapy.

The primary endpoint of the present trial was complete response, which has used in most clinical antiemetic trials. The "no emesis" represented the control of emesis, while "no use of rescue therapy" as an alternative marker also reflected control of nausea to a certain degree. In the present trial, only 5 patients experienced grade 1 to 2 emesis, and one patient with severe nausea received rescue treatment. Prior prospective studies on $\mathrm{NK}_{1} \mathrm{RAs}$ for prophylaxis of C-RINV observed that the $\mathrm{CR}$ rate was between $48 \%$ and $76 \%{ }^{14-16,18}$. As against the data of the above studies, our results exhibit that the triple antiemetic regimen achieved excellent antiemetic efficacy. One possible explanation is that the 5-day antiemetics administration increased the cumulative dose of the aprepitant and dexamethasone under the premise of multiple-day cisplatin chemotherapy, potentially having a stronger antiemetic effect. With regard to the secondary endpoints, $\mathrm{CP}$ is also a reliable endpoint to evaluate the overall control of nausea and vomiting, often being employed in clinical trials of CINV. The CP rate of this study achieved $72 \%$, which was higher than the data reported in several trials on aprepitant for prevention of CINV 24, 25 . However, the concept of "mild nausea" is not objective enough, whether it is based on grade 1 of CTCAE or NVAS $<25 \mathrm{~mm}^{26}$. Conversely, "no vomiting" and "no nausea" are relatively objective indicators, being adopted as secondary endpoints in our study. In the overall phase, $88.4 \%$ of patients developed no emesis, higher than the $66 \%-73 \%$ reported in previous studies on $\mathrm{NK}_{1}$ RAs for prevention of C-RINV 15,17 , 18,27 . Although the overall nausea-free response rate was only $60.5 \%$, this is approximately consistent with the $40 \%-61.5 \%$ reported in preceding research on $\mathrm{NK}_{1}$ RAs for prevention of C-RINV $16,18,27$.

The control rates of nausea and vomiting under our triple antiemetic regimen were also superior to the data reported in previous studies on concurrent chemoradiotherapy for head and neck squamous cell carcinoma. In actuality, many studies have reported treatment-related toxicities such as nausea and vomiting while publishing primary endpoints, yet few studies specified the antiemetic regimens. In the RTOG 0129 trial ${ }^{11}, 361$ patients were subjected to standard fractionation irradiation with a median dose of $69.8 \mathrm{~Gy}$ and concomitant triweekly cisplatin chemotherapy, and granisetron or ondansetron was used as the antiemetic regimen. In this condition, $65.9 \%$ of patients developed nausea and $46.8 \%$ of patients experienced vomiting. Further, the incidences of grade 3 nausea and grade 3-4vomiting were both close to $20 \%$. These results implied that single-agent $5-\mathrm{HT}_{3} \mathrm{RAs}$ were insufficient to control nausea and vomiting caused by concurrent chemoradiotherapy in head and neck squamous cell carcinoma. In a randomized phase 3 trial ${ }^{13}$, the CCRT arm was subjected to radical radiotherapy (66-70Gy) with concurrent weekly cisplatin $\left(30 \mathrm{mg} / \mathrm{m}^{2}\right)$. Employing ondansetron and dexamethasone as antiemetics, the overall incidences of nausea and vomiting were $47.7 \%$ and $30 \%$, and the incidences of grade 3 nausea and grade $3-4$ 
vomiting were only $1 \%$ and $1.5 \%$. As well as the addition of dexamethasone, the reduction of nausea and vomiting could also be attributed to the weekly cisplatin chemotherapy regimen. A preceding metaanalysis compared weekly low-dose $\left(\leq 50 \mathrm{mg} / \mathrm{m}^{2}\right)$ and triweekly high-dose cisplatin $\left(100 \mathrm{mg} / \mathrm{m}^{2}\right)$ for CCRT in LA-HNSCC, and demonstrated the weekly regimen did lead to a substantially lower proportion of severe nausea and/or vomiting (3\% vs $16 \%{ }^{28}$. However, the antiemetic efficacy was still superior to the above study under the premise of administrating the stronger emetic triweekly cisplatin regimen in the present study, illustrating that the addition of aprepitant into ondansetron and dexamethasone could more effectively control nausea and vomiting. In a further randomized phase 3 trial ${ }^{29}$, Tang et al also divided $100 \mathrm{mg} / \mathrm{m}^{2}$ of cisplatin into three days, and the antiemetic regimen stipulated adding metoclopramide on the basis of ondansetron/ granisetron and dexamethasone. The proportion of patients with nausea and vomiting were both around $80 \%$, and the incidence of grade 3 nausea was only $9 \%$, but the incidence of grade $3-4$ vomiting was up to $18 \%$. Numerically, although the triple regimen containing metoclopramide could be more effective in preventing severe nausea, poor prevention of the overall occurrence of nausea and vomiting was demonstrated, in addition to a failure to effectively prevent severe vomiting. Conversely, the majority of patients with nausea presented mainly mild and moderate levels in the present study. Despite the determination of the degree of nausea being subjective, only one patient required rescue antiemetics, and no patient discontinued treatment due to nausea or vomiting, thereby objectively illustrating that the triple regimen containing aprepitant could effectively control the occurrence of severe nausea and vomiting.

Recently, another prospective study evaluating the antiemetic efficacy of aprepitant in patients with LAHNSCC receiving radiotherapy and concurrent weekly cisplatin $\left(50 \mathrm{mg} / \mathrm{m}^{2}\right)$ chemotherapy was exhibited at the 2020 Annual Meeting of the American Society of Clinical Oncology (ASCO) ${ }^{27}$. The incidences of no nausea and no vomiting were $57.7 \%$ and $73.2 \%$ in patients receiving aprepitant, $5-\mathrm{HT}_{3} \mathrm{RA}$ and steroids. The similar antiemetic regimen demonstrated poorer control of nausea and vomiting during concurrent weekly cisplatin and radiation compared with our study. In general, whether horizontally compared with the studies of $\mathrm{NK}_{1}$ RAs for prophylaxis of C-RINV, or longitudinally compared with prior studies of CCRT for head and neck squamous cell carcinoma, our study administrated a triple antiemetic regimen consisting of aprepitant, ondansetron and dexamethasone and presented high antiemetic efficacy. Yet, the incidence of nausea in our study notably remained close to $40 \%$. A small randomized controlled trial had revealed that the addition of olanzapine increased the control rate of nausea from $40-71 \%{ }^{16}$. In future research, we will consider adding olanzapine to optimise this antiemetic regimen.

In terms of treatment compliance and toxicities, $86.0 \%$ of patients completed both cycles of chemotherapy in the present study. Of the six patients who discontinued chemotherapy, only three did so as a result of treatment related adverse events. Owing to the antiemetics that were given together with cisplatin, it was difficult to classify adverse events into antiemetics-related or cisplatin-related, especially fatigue, appetite loss and hepatic dysfunction. Nevertheless, constipation and hiccups with incidences of $65.1 \%$ and $16.3 \%$ were primarily regarded as antiemetics-related adverse events. The high incidence of constipation could be attributed to an increase of cumulative dose for 5-day administration of 
antiemetics, in addition to the non-use of prophylactic laxatives. Dexamethasone-induced hiccups are not an uncommon symptom in patients with cancer, Vardy et al. reported a $25 \%$ incidence of hiccups after dexamethasone administration ${ }^{30}$. Liaw et al. also discovered that more than $40 \%$ of patients treated with cisplatin developed hiccups, and $90 \%$ of hiccups eased after discontinuation of dexamethasone ${ }^{31}$. As against the data of the above studies, the incidence of hiccups in the present study was not unacceptable. A randomized trial confirmed that replacing dexamethasone with methylprednisolone does not compromise the antiemetic efficacy, but reduces the occurrence of hiccups. Hence, if there is a concern that hiccups would affect the quality of life, using methylprednisolone instead of dexamethasone could also be considered ${ }^{32}$. Although grade 3 mucositis and leucopenia also exceeded $10 \%$, both toxicities were recognised as being related to concurrent chemoradiotherapy. Overall, the present study demonstrated that the triple regimen is still well tolerated in HNSCC patients.

Notwithstanding the above, several limitations still exist in the present study. Firstly, 3-day administration of cisplatin was primarily owing to the naïve population tolerance and the recommendation of the local ethics committee, which had already been utilised in another large prospective trial ${ }^{29}$. The 5 -day antiemetic regimen in the present study was designed based on the 3-day administration of cisplatin. Thus, for the more extensively used single-day cisplatin administration, the question of whether this triple antiemetic regimen can achieve the same efficacy as the present study requires further research to answer. Further, the present study was a single-arm phase 2 trial without an optimal control group. When analysing the efficacy, only comparison with historical data was possible. For this reason, further phase 3 randomized controlled trials must be conducted in the future.

\section{Conclusions}

The present study extends the combination of aprepitant, ondansetron and dexamethasone as antiemetic prophylaxis to patients with HNSCC receiving IMRT and high-dose cisplatin chemotherapy. The addition of aprepitant into ondansetron and dexamethasone provides effective protection from emesis and nausea in patients with LA-HNSCC receiving radiotherapy and concomitant high-dose cisplatin chemotherapy. Treatment-related toxicities are mainly mild to moderate and tolerable. Randomized phase $\nabla$ studies are required to further define the potential role of $\mathrm{NK}_{1} \mathrm{RAs}$ in a chemoradiotherapy setting for HNSCC.

\section{List Of Abbreviations}

CINV, chemotherapy-induced nausea and vomiting; NK1RA, neurokinin-1 receptor antagonist; HEC, highly emetogenic chemotherapy; MASCC /ESMO, Multinational Association of Supportive Care in Cancer/ European Society for Medical Oncology; RINV, radiation-induced nausea and vomiting; LA-HNSCC, locally advanced head and neck squamous cell carcinoma; CCRT, concurrent chemoradiotherapy; C-RINV, chemoradiotherapy-induced nausea and vomiting; AJCC 7th, American Joint Committee on Cancer 7th Edition; ECOG, Eastern Cooperative Oncology Group; MDT, multi-disciplinary team; IMRT, intensity- 
modulated radiotherapy; $\mathrm{NCI}$ CTCAE, National Cancer Institute Common Toxicity Criteria for Adverse Events; MAT, MASCC Antiemesis Tool; FLIE, Functional Living Index-Emesis; EORTC, European

Organization for Research and Treatment of Cancer; QLQ-C30, Quality of Life Core 30 items; QLQ-H\&N35, Quality of Life Head and Neck 35 items; CR, complete response; CP, complete protection; NVAS, nausea visual analog scale; ASCO, American Society of Clinical Oncology.

\section{Declarations}

Ethics approval and consent to participate

This study was performed in line with the principles of the Declaration of Helsinki. Approval was granted by the Ethics Committee of National Cancer Center/Cancer Hospital, Chinese Academy of Medical Sciences and Peking Union Medical College (Approval No.17-115/1371).

Consent for publication

Not applicable.

Availability of data and materials

The dataset supporting the conclusions of this article is included within the article.

Competing interests

The authors have declared no conflicts of interest.

Funding

This work was supported by Beijing Hope Run Special Fund of Cancer Foundation of China, LC2017B17.

Preliminary results presented as oral presentation at the 62nd Annual Meeting of the American Society of Radiation Oncology (ASTRO), Virtual Meeting, Oct 25-28, 2020, and had been selected as one of "Best of ASTRO" abstract.

\section{Authors' contributions}

Zekun Wang: data analysis and interpretation, manuscript preparation and editing.

Junlin Yi, Ye Zhang: conception and design, quality control of data and algorithms, manuscript review and final approval.

Wenyang Liu, Jianghu Zhang, Xuesong Chen, Jingbo Wang, Kai Wang, Yuan Qu, Xiaodong Huang, Jingwei Luo, Jianping Xiao, Guozhen. Xu, Li Gao: data acquisition and manuscript review and final approval. 


\section{Acknowledgements}

We acknowledge our families and all the patients.

\section{References}

1. Navari RM, Aapro M. Antiemetic Prophylaxis for Chemotherapy-Induced Nausea and Vomiting. $N$ Engl J Med. Apr 7 2016;374(14):1356-67. doi:10.1056/NEJMra1515442

2. Herrstedt J, Roila F, Warr D, et al. 2016 Updated MASCC/ESMO Consensus Recommendations: Prevention of Nausea and Vomiting Following High Emetic Risk Chemotherapy. Support Care Cancer. Jan 2017;25(1):277-288. doi:10.1007/s00520-016-3313-0

3. Razvi Y, Chan S, McFarlane T, et al. ASCO, NCCN, MASCC/ESMO: a comparison of antiemetic guidelines for the treatment of chemotherapy-induced nausea and vomiting in adult patients. Support Care Cancer. Jan 2019;27(1):87-95. doi:10.1007/s00520-018-4464-y

4. Feyer P, Jahn F, Jordan K. Prophylactic Management of Radiation-Induced Nausea and Vomiting. Biomed Res Int. 2015;2015:893013. doi:10.1155/2015/893013

5. Enblom A, Bergius Axelsson B, Steineck G, Hammar M, Borjeson S. One third of patients with radiotherapy-induced nausea consider their antiemetic treatment insufficient. Support Care Cancer. Jan 2009;17(1):23-32. doi:10.1007/s00520-008-0445-x

6. Ruhlmann $\mathrm{CH}$, Herrstedt J. New treatments on the horizon for chemoradiotherapy-induced nausea and vomiting. Expert Opin Pharmacother. Aug 2016;17(12):1623-9. doi:10.1080/14656566.2016.1202923

7. Fraunholz I, Grau K, Weiss C, Rodel C. Patient- and treatment-related risk factors for nausea and emesis during concurrent chemoradiotherapy. Strahlenther Onkol. Jan 2011;187(1):1-6. doi:10.1007/s00066-010-2196-0

8. Maranzano E, De Angelis V, Pergolizzi S, et al. A prospective observational trial on emesis in radiotherapy: analysis of 1020 patients recruited in 45 Italian radiation oncology centres. Radiother Oncol. Jan 2010;94(1):36-41. doi:10.1016/j.radonc.2009.11.001

9. Grunberg S, Chua D, Maru A, et al. Single-dose fosaprepitant for the prevention of chemotherapyinduced nausea and vomiting associated with cisplatin therapy: randomized, double-blind study protocol--EASE. J Clin Oncol. Apr 10 2011;29(11):1495-501. doi:10.1200/JC0.2010.31.7859

10. Ang KK, Zhang Q, Rosenthal DI, et al. Randomized phase III trial of concurrent accelerated radiation plus cisplatin with or without cetuximab for stage III to IV head and neck carcinoma: RTOG 0522. J Clin Oncol. Sep 20 2014;32(27):2940-50. doi:10.1200/JC0.2013.53.5633

11. Nguyen-Tan PF, Zhang Q, Ang KK, et al. Randomized phase III trial to test accelerated versus standard fractionation in combination with concurrent cisplatin for head and neck carcinomas in the Radiation Therapy Oncology Group 0129 trial: long-term report of efficacy and toxicity. J Clin Oncol. Dec 1 2014;32(34):3858-66. doi:10.1200/JC0.2014.55.3925 
12. Noronha V, Joshi A, Patil VM, et al. Once-a-Week Versus Once-Every-3-Weeks Cisplatin Chemoradiation for Locally Advanced Head and Neck Cancer: A Phase III Randomized Noninferiority Trial. J Clin Oncol. Apr 10 2018;36(11):1064-1072. doi:10.1200/jco.2017.74.9457

13. Patil VM, Noronha $V$, Joshi A, et al. A randomized phase 3 trial comparing nimotuzumab plus cisplatin chemoradiotherapy versus cisplatin chemoradiotherapy alone in locally advanced head and neck cancer. Cancer. Sep 15 2019;125(18):3184-3197. doi:10.1002/cncr.32179

14. Jahn F, Riesner A, Jahn P, Sieker F, Vordermark D, Jordan K. Addition of the Neurokinin-1-Receptor Antagonist (RA) Aprepitant to a 5-Hydroxytryptamine-RA and Dexamethasone in the Prophylaxis of Nausea and Vomiting Due to Radiation Therapy With Concomitant Cisplatin. Int J Radiat Oncol Biol Phys. Aug 1 2015;92(5):1101-1107. doi:10.1016/j.jjrobp.2015.04.037

15. Hanawa S, Mitsuhashi A, Matsuoka A, et al. Efficacy of palonosetron plus aprepitant in preventing chemoradiotherapy-induced nausea and emesis in patients receiving daily low-dose cisplatin-based concurrent chemoradiotherapy for uterine cervical cancer: a phase II study. Support Care Cancer. Nov 2016;24(11):4633-8. doi:10.1007/s00520-016-3306-z

16. Navari RM, Nagy CK, Le-Rademacher J, Loprinzi CL. Olanzapine versus fosaprepitant for the prevention of concurrent chemotherapy radiotherapy-induced nausea and vomiting. J Community Support Oncol. Apr 2016;14(4):141-7. doi:10.12788/jcso.0245

17. Ruhlmann $\mathrm{CH}$, Christensen TB, Dohn LH, et al. Efficacy and safety of fosaprepitant for the prevention of nausea and emesis during 5 weeks of chemoradiotherapy for cervical cancer (the GAND-emesis study): a multinational, randomised, placebo-controlled, double-blind, phase 3 trial. The Lancet Oncology. 2016;17(4):509-518. doi:10.1016/s1470-2045(15)00615-4

18. Ades S, Halyard M, Wilson K, et al. Effectiveness of aprepitant in addition to ondansetron in the prevention of nausea and vomiting caused by fractionated radiotherapy to the upper abdomen (AVERT). Support Care Cancer. May 2017;25(5):1503-1510. doi:10.1007/s00520-016-3540-4

19. Yi J, Huang X, Gao L, et al. Intensity-modulated radiotherapy with simultaneous integrated boost for locoregionally advanced nasopharyngeal carcinoma. Radiat Oncol. Feb 18 2014;9:56. doi:10.1186/1748-717x-9-56

20. Brouwer CL, Steenbakkers RJHM, Bourhis J, et al. CT-based delineation of organs at risk in the head and neck region: DAHANCA, EORTC, GORTEC, HKNPCSG, NCIC CTG, NCRI, NRG Oncology and TROG consensus guidelines. Radiotherapy and Oncology. 2015;117(1):83-90.

doi:10.1016/j.radonc.2015.07.041

21. Einhorn LH, Rapoport B, Navari RM, Herrstedt J, Brames MJ. 2016 updated MASCC/ESMO consensus recommendations: prevention of nausea and vomiting following multiple-day chemotherapy, high-dose chemotherapy, and breakthrough nausea and vomiting. Support Care Cancer. Jan 2017;25(1):303-308. doi:10.1007/s00520-016-3449-y

22. Englert S, Kieser M. Adaptive designs for single-arm phase II trials in oncology. Pharm Stat. May-Jun 2012;11(3):241-9. doi:10.1002/pst.541 
23. Kawaguchi R, Tanase Y, Haruta S, Yoshida S, Furukawa N, Kobayashi H. Addition of aprepitant to standard therapy for prevention of nausea and vomiting among patients with cervical cancer undergoing concurrent chemoradiotherapy. Int J Gynaecol Obstet. Dec 2015;131(3):312-3. doi:10.1016/j.jjgo.2015.05.030

24. Hesketh PJ, Grunberg SM, Gralla RJ, et al. The oral neurokinin-1 antagonist aprepitant for the prevention of chemotherapy-induced nausea and vomiting: a multinational, randomized, doubleblind, placebo-controlled trial in patients receiving high-dose cisplatin--the Aprepitant Protocol 052 Study Group. J Clin Oncol. Nov 15 2003;21(22):4112-9. doi:10.1200/JC0.2003.01.095

25. Poli-Bigelli S, Rodrigues-Pereira J, Carides AD, et al. Addition of the neurokinin 1 receptor antagonist aprepitant to standard antiemetic therapy improves control of chemotherapy-induced nausea and vomiting. Results from a randomized, double-blind, placebo-controlled trial in Latin America. Cancer. Jun 15 2003;97(12):3090-8. doi:10.1002/cncr.11433

26. Bosnjak SM, Gralla RJ, Schwartzberg L. Prevention of chemotherapy-induced nausea: the role of neurokinin-1 (NK1) receptor antagonists. Support Care Cancer. May 2017;25(5):1661-1671. doi:10.1007/s00520-017-3585-z

27. D'souza H, Noronha V, Patil VM, Joshi A, Prabhash K. Aprepitant in weekly cisplatin with radiation in head and neck cancer: Is it required? Journal of Clinical Oncology. 2020;38(15_suppl):e24122e24122.

28. Szturz P, Wouters K, Kiyota N, et al. Weekly Low-Dose Versus Three-Weekly High-Dose Cisplatin for Concurrent Chemoradiation in Locoregionally Advanced Non-Nasopharyngeal Head and Neck Cancer: A Systematic Review and Meta-Analysis of Aggregate Data. Oncologist. Sep 2017;22(9):1056-1066. doi:10.1634/theoncologist.2017-0015

29. Tang $L-Q$, Chen $D-P, G$ uo $L$, et al. Concurrent chemoradiotherapy with nedaplatin versus cisplatin in stage II-IVB nasopharyngeal carcinoma: an open-label, non-inferiority, randomised phase 3 trial. The Lancet Oncology. 2018;19(4):461-473. doi:10.1016/s1470-2045(18)30104-9

30. Vardy J, Chiew KS, Galica J, Pond GR, Tannock IF. Side effects associated with the use of dexamethasone for prophylaxis of delayed emesis after moderately emetogenic chemotherapy. $\mathrm{Br} \mathrm{J}$ Cancer. Apr 10 2006;94(7):1011-5. doi:10.1038/sj.bjc.6603048

31. Liaw $\mathrm{CC}$, Wang $\mathrm{CH}$, Chang HK, et al. Cisplatin-related hiccups: male predominance, induction by dexamethasone, and protection against nausea and vomiting. $J$ Pain Symptom Manage. Oct 2005;30(4):359-66. doi:10.1016/j.jpainsymman.2005.08.008

32. Go SI, Koo DH, Kim ST, et al. Antiemetic Corticosteroid Rotation from Dexamethasone to Methylprednisolone to Prevent Dexamethasone-Induced Hiccup in Cancer Patients Treated with Chemotherapy: A Randomized, Single-Blind, Crossover Phase III Trial. Oncologist. Nov 2017;22(11):1354-1361. doi:10.1634/theoncologist.2017-0129

\section{Figures}


$\begin{array}{lllllll}\text { Week } 1 & \text { Week } 2 & \text { Week } 3 & \text { Week } 4 & \text { Week } 5 & \text { Week } 6 & \text { Week } 7\end{array}$

IMRT

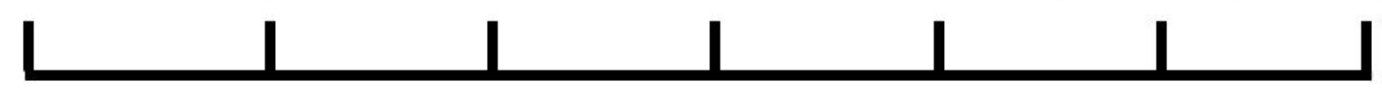
$100 \mathrm{mg} / \mathrm{m}^{2} / 3$ days iv
$100 \mathrm{mg} / \mathrm{m}^{2} / 3$ days iv
Cisplatin
I I I
I I I
$125 \mathrm{mg} \mathrm{d} 1,80 \mathrm{mg}$ d2 -5 po
Aprepitant

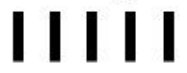
$125 \mathrm{mg}$ d22, $80 \mathrm{mg}$ d23-26 po
| | | | |
$8 \mathrm{mg} \mathrm{d} 1$ iv
Ondansetron
$12 \mathrm{mg} \mathrm{d} 1,8 \mathrm{mg}$ d2-5 po
Dexamethasone | | | | |
$12 \mathrm{mg}$ d22, $8 \mathrm{mg}$ d23-26 po
| I I | |

Week 1 Week 2 Week 3 Week 4 Week 5 Week 6 Week 7

QOL scale

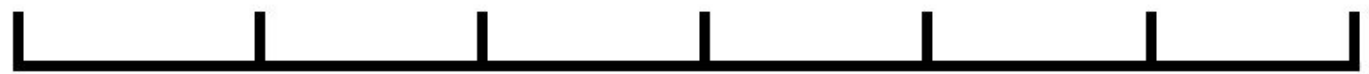

Figure 1

The trial procedure Abbreviation: IMRT: Intensity-modulated radiotherapy; QOL: Quality of Life

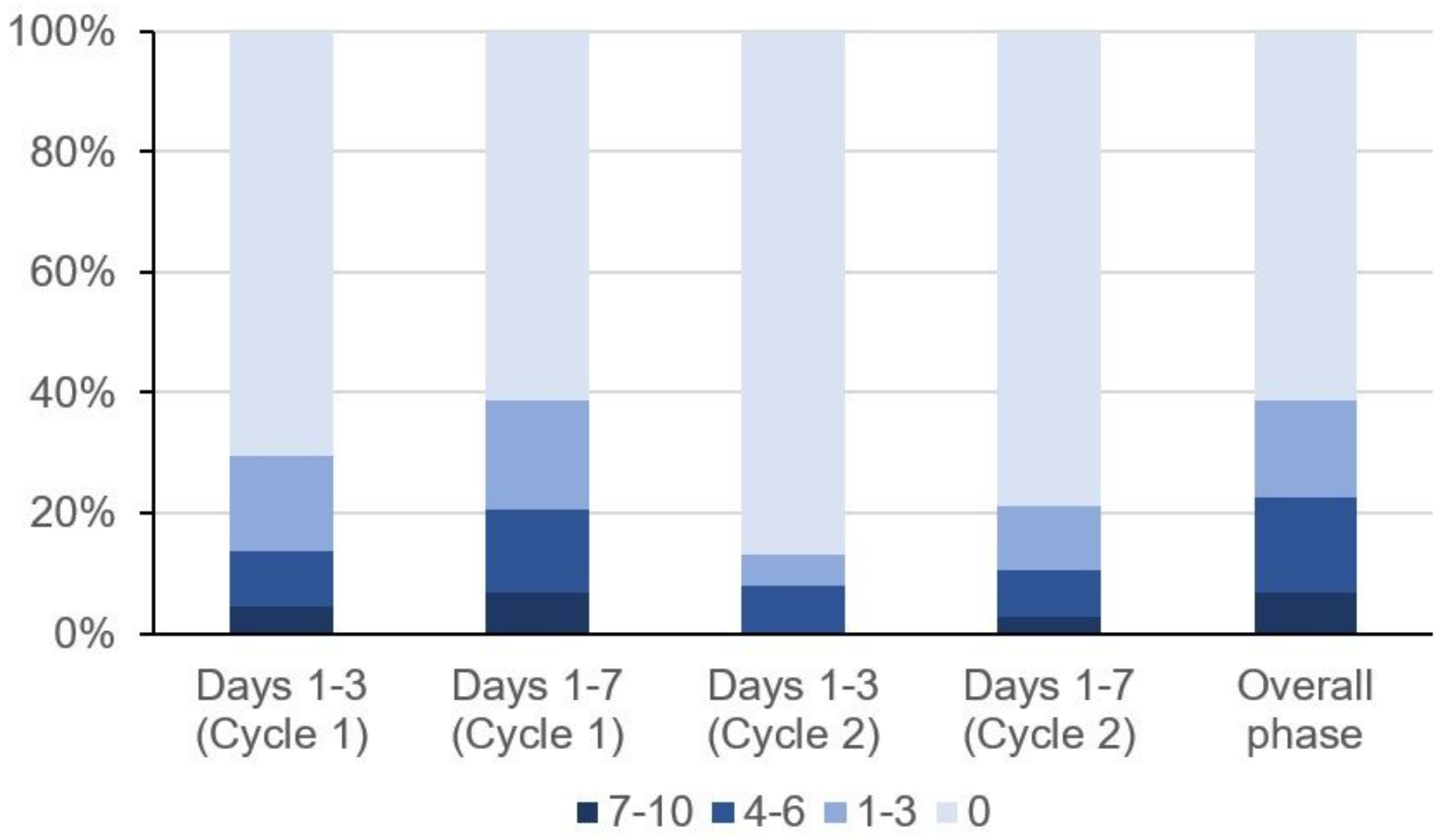

Figure 2 
The distribution of patients with different grades of nausea based on the NVAS score 